\title{
Plasma wave observation using waveform capture in the Lunar Radar Sounder on board the SELENE spacecraft
}

\author{
Yoshiya Kasahara ${ }^{1}$, Yoshitaka Goto ${ }^{1}$, Kozo Hashimoto ${ }^{2}$, Tomohiko Imachi ${ }^{1}$, \\ Atsushi Kumamoto ${ }^{3}$, Takayuki Ono ${ }^{3}$, and Hiroshi Matsumoto ${ }^{4}$ \\ ${ }^{1}$ Kanazawa University, Kanazawa 920-1192, Japan \\ ${ }^{2}$ Kyoto University, Uji 611-0011, Japan \\ ${ }^{3}$ Tohoku University, Sendai 980-8578, Japan \\ ${ }^{4}$ Kyoto University, Kyoto 606-8501, Japan
}

(Received March 28, 2007; Revised July 24, 2007; Accepted August 14, 2007; Online published April 9, 2008)

\begin{abstract}
The waveform capture (WFC) instrument is one of the subsystems of the Lunar Radar Sounder (LRS) on board the SELENE spacecraft. By taking advantage of a moon orbiter, the WFC is expected to measure plasma waves and radio emissions that are generated around the moon and/or that originated from the sun and from the earth and other planets. It is a high-performance and multifunctional software receiver in which most functions are realized by the onboard software implemented in a digital signal processor (DSP). The WFC consists of a fastsweep frequency analyzer (WFC-H) covering the frequency range from $1 \mathrm{kHz}$ to $1 \mathrm{MHz}$ and a waveform receiver (WFC-L) in the frequency range from $10 \mathrm{~Hz}$ to $100 \mathrm{kHz}$. By introducing the hybrid IC called PDC in the WFC-H, we created a spectral analyzer with a very high time and frequency resolution. In addition, new techniques such as digital filtering, automatic filter selection, and data compression are implemented for data processing of the WFC-L to extract the important data adequately under the severe restriction of total amount of telemetry data. Because of the flexibility of the instruments, various kinds of observation modes can be achieved, and we expect the WFC to generate many interesting data.
\end{abstract}

Key words: The SELENE project, waveform capture (WFC), software receiver, lunar wake, plasma wave, radio emission.

\section{Introduction}

The SELENE project is the first Japanese lunar exploration mission. The craft will explore the surface distribution of elements and minerals, surface and subsurface structures of the moon, the gravitational field, the magnetic field, and energy particles originating from the moon, as well as from solar/interplanetary space (SELENE Project, 2007). The SELENE spacecraft consists of the main orbiter and two small orbiters. The Lunar Radar Sounder (LRS) is one of the scientific instruments on board the SELENE main orbiter (Ono et al., 2008). The LRS consists of three subsystems: the sounder observation (SDR), the natural plasma wave receiver (NPW), and the waveform capture (WFC). A main objective of the SDR is to investigate the surface and subsurface structures of the moon using an HF radar technique, and the NPW and the WFC are designed to measure natural plasma waves around the moon and in interplanetary space originating from the sun, from the earth, and from other planets. The NPW covers the frequency range from $20 \mathrm{kHz}$ to $30 \mathrm{MHz}$, and the WFC covers the lower frequency range from $100 \mathrm{~Hz}$ to $1 \mathrm{MHz}$.

The WFC is a software receiver (Hashimoto et al., 2003) in which most of the functions are realized by a digital signal processor (DSP) and programmable down convert-

Copyright (C) The Society of Geomagnetism and Earth, Planetary and Space Sciences (SGEPSS); The Seismological Society of Japan; The Volcanological Society of Japan; The Geodetic Society of Japan; The Japanese Society for Planetary Sciences; TERRAPUB. ers (PDC) implemented on the WFC board. The amount of raw data from the plasma wave instrument is huge because the scientific objectives require the covering of a wide frequency range with high time and frequency resolution; furthermore, a variety of operation modes are needed to meet these scientific objectives. It is quite difficult to satisfy these requirements with only analogue circuits because of the severe restrictions of power consumption, weight, and size of the instruments. Digital signal processing onboard the spacecraft is useful for the plasma wave measurements because a variety of operational modes can be implemented in the DSP's onboard software without changing the hardware configuration. This kind of digital receiver was designed, for example, for the low-frequency receiver (LFA) on board the NOZOMI spacecraft (Matsumoto et al., 1998). This technique was further developed for the plasma wave analyzer (PWA) for the SS-520-2 rocket experiment (Hashimoto et al., 2003). In the development process of the onboard software for the WFC onboard the SELENE spacecraft, we have additionally implemented several techniques of digital signal processing to extract important data for each scientific objective by adequately changing the observation mode and reducing the total amount of telemetry data. In the present paper, we introduce an overview of the WFC onboard the SELENE spacecraft. 


\section{Scientific Objectives}

The WFC on board SELENE observes plasma waves from $100 \mathrm{~Hz}$ to $1 \mathrm{MHz}$ at about 60 of earth's radii $\left(R_{\mathrm{E}}\right)$ in the solar wind, in the magnetosphere, and sometimes in the lunar wake. Joint observations with other spacecraft also play an important role. The scientific objectives of the WFC are roughly fall into two categories: 1) lunar science and 2) extra-lunar science. The former is the science of the plasma physics related to the moon itself. The latter is the science of the plasma and radio waves originating from the sun and the earth and other planets, taking advantage of a moon orbiter.

As for science on the moon, one of the phenomena of most interest to be obtained from the WFC data is the dynamics of lunar wake as a result of solar wind-moon interaction. Many new systematic wake observations are expected, even though there have been several reports on plasma wave observations related to the lunar wake during flybys. The WIND spacecraft passed through the solar wind wake of the moon at a distance of $\sim 6.8$ lunar radii $\left(R_{\mathrm{L}}\right)$. Kellogg et al. (1996) introduced several different wave modes. They showed that the frequency of locally generated Langmuir waves fell to $4 \mathrm{kHz}$, which was the lower frequency limit of the TNR (Bougeret et al., 1995) onboard WIND, because of the lunar wake vacuum, but the frequency fluctuated considerably, suggesting turbulence of the wake boundary. Kel$\log$ et al. (1996) also showed a descending tone in the frequency range of 100 to $50 \mathrm{~Hz}$ obtained from the search coils onboard WIND. This wave was identified as a whistler mode signal in the wake region. WIND observed other interesting waves both inside and outside the wake that were not certainly indentifiable with modes of a uniform plasma, and it is suggested that these waves are associated with density discontinuity and a velocity shear at the boundary of the wake (Kellogg et al., 1996). Farrell et al. (1997) demonstrated that ion beams generated by an ambipolar electric field near the wake flanks are capable of generating broadband electrostatic noise that was observed in the frequency range of up to $6 \mathrm{kHz}$ by measured by WIND.

While WIND crossed the lunar wake at a distance of $\sim 6.8 R_{\mathrm{L}}$, SELENE will orbit the moon at an altitude of $100 \mathrm{~km}$, and the encounter with the lunar wake will frequently occur in a region much closer to the moon. The nature of the plasma wave caused by the lunar wake at such a low-altitude region is not known at all. As for the theoretical study or computer simulation on the lunar wake, Trávníček et al. (2005) studied the structure and properties of the lunar wake using two-dimensional global hybrid simulations and suggested that the downstream region of the lunar wake is dominated by electromagnetic turbulence with frequencies near the local proton gyrofrequency for given solar wind conditions. But their interest in the work was mainly on the global structure of the lunar wake in the scale length of a few tens of $R_{\mathrm{L}}$, and the detailed structure of the lunar wake near the moon was not discussed.

Another scientific topic in the field of lunar plasma physics concerns the minimagnetosphere caused by the magnetic anomaly of the moon. The moon is basically known as a nonmagnetic satellite, but there are several regions in which relatively strong magnetic anomalies exist.
For example, Kurata et al. (2005) suggested the presence of a minimagnetosphere over the Reiner Gamma magnetic anomaly (RGA) region on the moon. They estimated the vertical size of the minimagnetosphere to be about $30 \mathrm{~km}$ or more. Kuncic et al. (2004) proposed a model for radio emissions associated with the minimagnetosphere on the moon and predicted that $f_{\mathrm{p}}$ and $2 f_{\mathrm{p}}$ emissions are radiated in the foreshock region of a shock front associated with the minimagnetosphere on the lunar surface.

Apart from the wave phenomena related to the solar wind-moon interaction, there are various kinds of plasma waves to be observed from the moon. In the history of Japanese scientific spacecraft, the GEOTAIL mission is one of the most important projects investigating the phenomena in the earth's magnetosphere, by which so many new findings and paradigms were introduced by combining observation, computer simulations and theoretical studies. The WFC onboard SELENE is expected to clarify the detailed features of the wave phenomena observed by PWI instruments (Matsumoto et al., 1994a) on board the GEOTAIL spacecraft, such as Auroral Kilometric Radiation (AKR) (Murata et al., 1997; Hashimoto et al., 1998), nonthermal continuum and kilometric continuum (KC) (Hashimoto et al., 1999, 2005, 2006), continuum enhancement (Kasaba et al., 1998), low-frequency bursts (Anderson et al., 1997), electrostatic solitary wave (ESW) (Matsumoto et al., 1994b), narrowband electrostatic noise (NEN) (Kojima et al., 2001), $f_{\mathrm{p}}$ and $2 f_{\mathrm{p}}$ emissions (Kasaba et al., 2000), and solar radio emissions (Kasahara et al., 2001), among others. Emissions with frequencies higher than the maximum plasma frequency of the magnetosheath (about $30 \mathrm{kHz}$ ), such as AKR, KC, and continuum enhancement, and type II and III solar bursts are observed both inside and outside the magnetosphere. The lower frequency emissions, such as ESW or NEN, cannot go through the magnetosheath. ESW was also observed in the solar wind by WIND (Mangeney et al., 1999). The nonthermal continuum has both trapped and untrapped components. The low-frequency bursts are much affected near the solar wind plasma frequency. Although there was a trial to observe Jovian emissions under the terrestrial emissions by the GEOTAIL spacecraft (Kasaba et al., 1996), SELENE is expected to observe planetary emissions when terrestrial emissions are blocked by the moon.

\section{Design of the WFC Instrument \\ 3.1 Electrical design}

Figure 1 shows a block diagram of the WFC. It measures two components of electric wave signals detected by the two orthogonal $30 \mathrm{~m}$ tip-to-tip antennas. The signals detected by these antennas are passed through the preamplifiers, which are commonly used with the other LRS subsystems, and fed into the main electronics of the WFC. The main electronics of the WFC consist of two boards; one is used for the analogue circuits, and the other for digital circuits. The size of each board is ISO A4. Power consumption of the WFC main electronics is $12.2 \sim 13.3 \mathrm{~W}$, depending on the operation mode. The detailed physical and electrical properties of the LRS system are described by Ono et al. (2008). Because SELENE is three-axes stabilized, it 


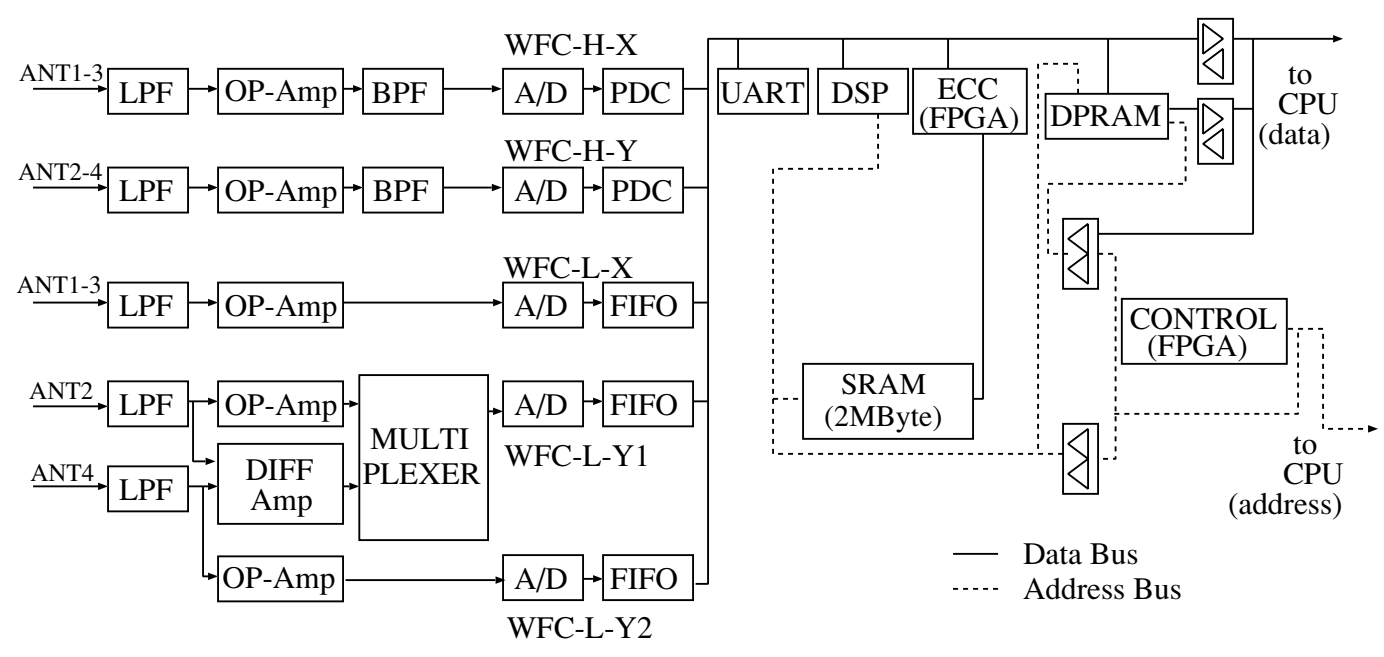

Fig. 1. A block diagram of the WFC.

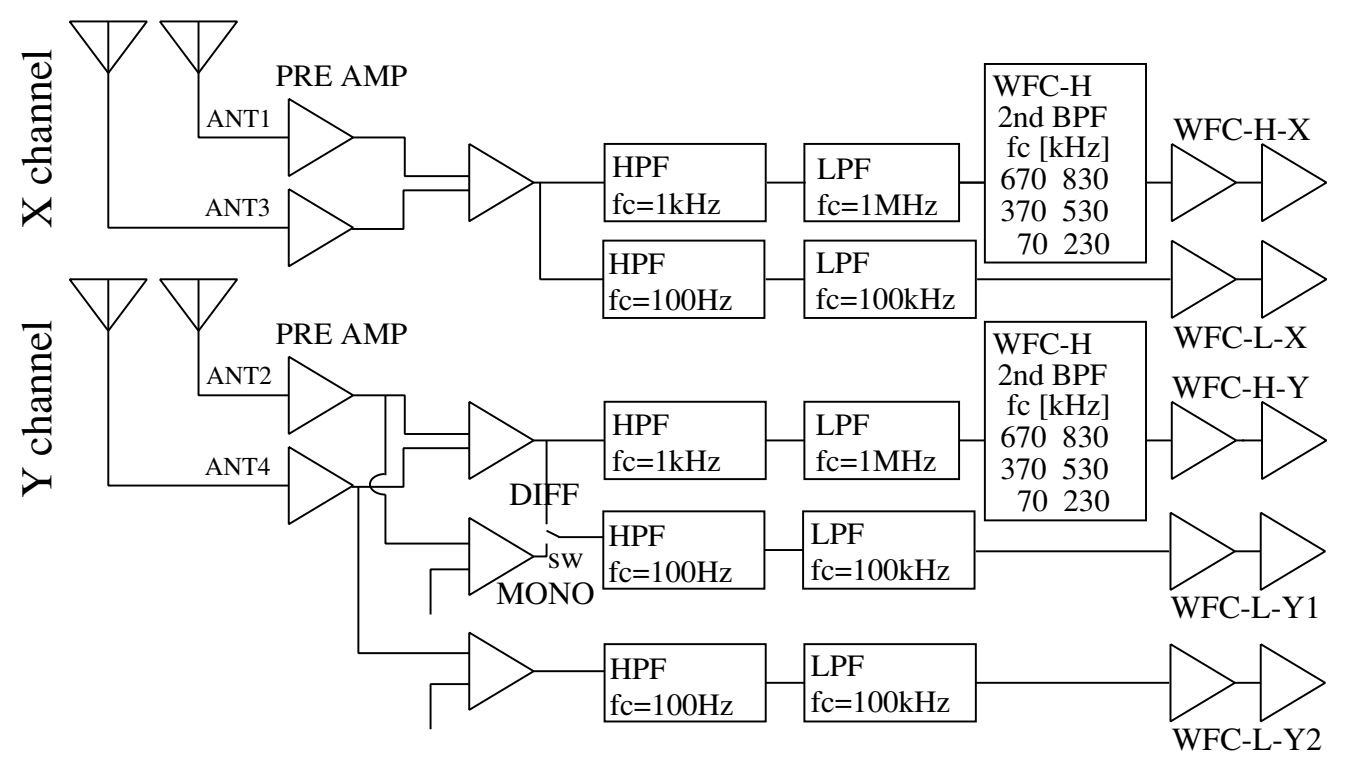

Fig. 2. A detailed block diagram of the WFC analogue board.

is important to measure the phase difference of the signals measured by the two pairs of antennas to determine the polarization of the wave. Therefore, the WFC is designed to obtain the exact timing of the wave signals to determine the precise phase difference between the two pairs.

The main electronic components of the WFC consist of two kinds of passive receivers named WFC-H and WFC-L. The WFC-H is a fast-sweep frequency analyzer covering the frequency range from $1 \mathrm{kHz}$ to $1 \mathrm{MHz}$. The WFC-L measures waveforms in the frequency range from $10 \mathrm{~Hz}$ to $100 \mathrm{kHz}$. As shown in Fig. 1, the WFC has five channels of signal input module; two are for the WFC-H (WFC-H$\mathrm{X}$ and WFC-H-Y), and the other three are for the WFC-L (WFC-L-X, WFC-L-Y1 and WFC-L-Y2). The signals fed to these five channels are passed through low-pass filters (LPF) and high-pass filters (HPF) and are independently converted to digital signals by the ADCs (analog-digital converters). On board the WFC, the AD9260, which is a 16bit, high-speed oversampled ADC (Analog Devices, 2000), is adopted for these five ADCs.
Figure 2 shows a detailed block diagram of the analogue circuits of the WFC. The signals from a pair of antennas named ANT1 and ANT3 are first passed through a differential amplifier and separated into the WFC-H-X and WFC-L-X signals. The signal for the WFC-H-X is then passed through an HPF and an LPF with cutoff frequencies of $1 \mathrm{kHz}$ and $1 \mathrm{MHz}$. There are three optional LPFs and three optional HPFs. The cutoff frequencies of the LPFs are $70 \mathrm{kHz}, 370 \mathrm{kHz}$, and $670 \mathrm{kHz}$, and those of the HPFs are $230 \mathrm{kHz}, 530 \mathrm{kHz}$, and $830 \mathrm{kHz}$, respectively. These filters can be assigned as "filter" or "through" by command, and thus the signal for the specified frequency band can be passed depending on the combination of these filters. If we set the modes of all filters as "through", the signal with a frequency range from $1 \mathrm{kHz}$ to $1 \mathrm{MHz}$ is then all passed through into the latter part of the circuits. In the last part of the circuits, two taps of gain amplifier are equipped, and we can select the gain of the signal from among $0 \mathrm{~dB}, 20 \mathrm{~dB}$, and $40 \mathrm{~dB}$.

The signal is then fed to the ADCs and oversampled at a 


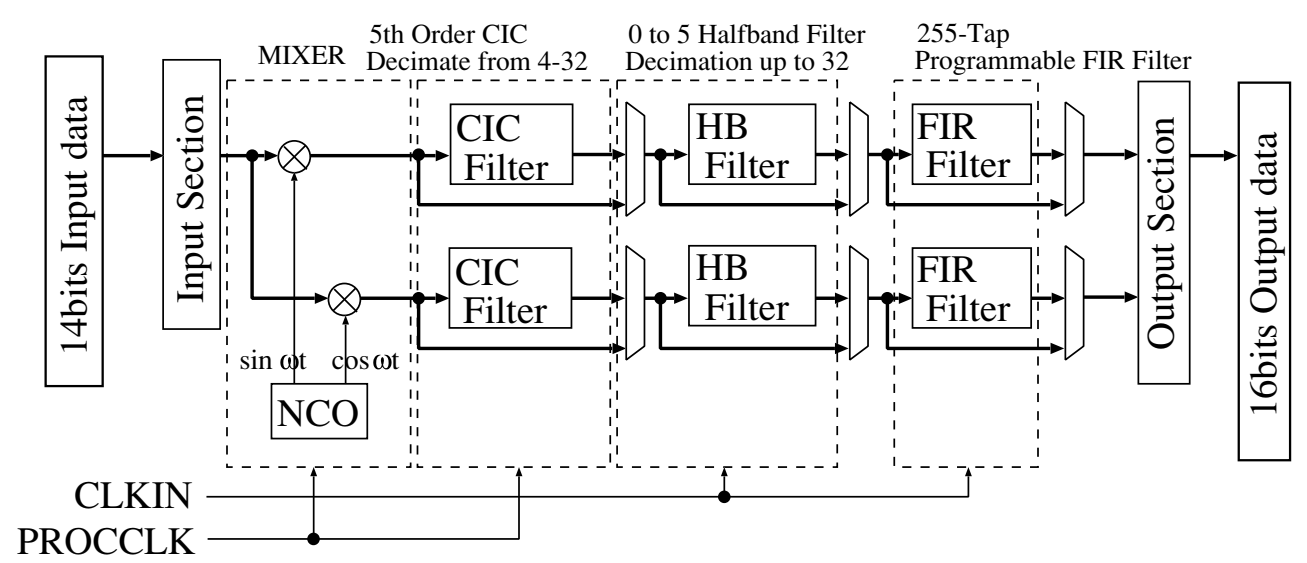

Fig. 3. A block diagram of the programmable down converter (PDC).

frequency of $20 \mathrm{MHz}$. The attenuation of the $1 \mathrm{MHz}$ LPF in the $\mathrm{ADC}$ at frequencies higher than $10 \mathrm{MHz}$ is more than $70 \mathrm{~dB}$. The output word rate $\left(f_{\mathrm{s}}\right)$ is $2.5 \mathrm{MHz}(8 \mathrm{x}$ oversampling) and fed to the PDC. The PDC converts the signal frequency $f$ to $f-f_{\mathrm{c}}$, where $f_{\mathrm{c}}$ is the local frequency and is swept, and a certain frequency range in the base band is Fourier analyzed. A detailed description is given in Sections 3.2 and 4.2. This system makes spectrum analysis possible in high time and frequency resolution. The optional LPFs and HPFs introduced above are prepared to avoid interference in the interested band during the sweep. The circuits for WFC-H-Y, which measures differential signal between another pair of antennas named ANT2 and ANT4, are the same as the ones for WFC-H-X.

On the other hand, there are three channels for the WFCL: WFC-L-X, WFC-L-Y1, and WFC-L-Y2. The signal fed to WFC-L-X is the differential signal between ANT1 and ANT3. As shown in Fig. 2, WFC-L-Y1 has two operation modes: differential and interferometry. In the former mode, the differential signal between ANT2 and ANT4 is fed to WFC-L-Y1. In the latter mode, we use ANT2 and ANT4 as a pair of monopole antennas. The signal from ANT2 is fed to WFC-L-Y1, and that from ANT4 is fed to WFC-L-Y2. This mode is dedicated to measuring the phase velocities and wave numbers of the observed plasma waves.

Each signal is then passed through an HPF and an LPF with their cutoff frequencies of $100 \mathrm{~Hz}$ and $100 \mathrm{kHz}$. Since the $100 \mathrm{~Hz} \mathrm{HPF}$ is a first-order RC filter and the intensities of low-frequency emissions increase inversely proportional to frequencies, the observable lowest frequency is virtually much lower. The signal is amplified by $0 \mathrm{~dB}, 20 \mathrm{~dB}$, or $40 \mathrm{~dB}$ with the two taps of gain amplifier, then $8 \mathrm{x}$ oversampled by the ADC with $2 \mathrm{MHz}$ or $1 \mathrm{MHz}$. The waveform in the frequency range below $100 \mathrm{kHz}$ with $f_{\mathrm{s}}=250 \mathrm{kHz}$ or $50 \mathrm{kHz}$ with $f_{\mathrm{s}}=125 \mathrm{kHz}$ is easily switched over by changing the clock frequency of the ADC by command. At $1 \mathrm{MHz}$ sampling, the WFC-L covers the frequency range below $50 \mathrm{kHz}$, and the attenuation of the $100 \mathrm{kHz}$ LPF at frequencies higher than $0.95 \mathrm{MHz}$ is more than $70 \mathrm{~dB}$ to suppress the aliasing from 0.95 to $1.05 \mathrm{MHz}$.

\subsection{Onboard data processing}

The signals digitized by the ADCs are processed by the PDCs and the DSP in the digital circuits of the WFC sub- system and sent to the onboard computer (OBC) through a DPRAM (Dual-Port RAM). As a DSP onboard the WFC, the SMJ320C31 (Texas Instruments, 2006) has been adopted. The OBC manages the whole operation of the LRS, and the WFC data submitted to the OBC are edited with the other subsystems of the LRS and sent to ground through the satellite telemetry. Because the telemetry budget is restricted compared to the amount of raw data, we need to process the raw data and reduce the amount of mission data to be sent to the ground by the DSP.

To perform spectral analysis with very high time and frequency resolution, the WFC-H has two hybrid ICs called PDCs (Programmable Down Converters). As was described in Introduction, it is quite difficult to construct a highperformance spectral analyzer with only analogue circuits because of the severe restrictions of power consumption, weight, and size of instruments. Conventional systems, such as GEOTAIL, use a multichannel analyzer (MCA) for higher time resolution with lower frequency resolution and a sweep frequency analyzer (SFA) for higher frequency resolution with lower time resolution (Matsumoto et al., 1994a). On the other hand, the computation load of the signal processing is still too heavy, even though we adopt the DSP if we process the digital signals directly from the ADCs with an output word rate of $2.5 \mathrm{MHz}$. The PDCs are used for converting the wide band input signals from antennas at a $2.5 \mathrm{MHz}$ word rate into narrow band signals and for down-sampling the data. The HSP50214BVI (Intersil Corporation, 2000) has been adopted as a PDC in the WFC-H. The block diagram of the PDC is shown in Fig. 3. Note that the input interface for the PDC is 14 bits in spite of the 16-bit resolution of the ADC, and the lower 2 bits of the output from the ADC are dismissed. The signal fed into the $\mathrm{PDC}$ is down-converted by digital mixers and a quadrature numerical controlled oscillator (NCO), and filtered and decimated by the following three decimation filters: cascaded integrator-comb (CIC), half-band (HB), and finite-impulse response (FIR). The PDCs are controlled by the DSP software, and the parameters used for the PDCs, such as frequency of the NCO, decimation rate, and bandwidth of the decimation filters, can be quickly changed. We can then get the in-phase and quadrature components of the downsampled data from the PDC. The output data from the PDC 
Table 1. Frequency bands and frequency resolutions of the WFC-H.

(a) L-mode

\begin{tabular}{ccc}
\hline Band No. & Freq. band $[\mathrm{kHz}]$ & $\Delta f[\mathrm{~Hz}]$ \\
\hline 0 & $0.00 \sim 9.77$ & 38.2
\end{tabular}

(b) H-mode

\begin{tabular}{|c|c|c|c|c|c|}
\hline Band No. & Freq. band $[\mathrm{kHz}]$ & $\Delta f[\mathrm{kHz}]$ & Band No. & Freq. band $[\mathrm{kHz}]$ & $\Delta f[\mathrm{kHz}]$ \\
\hline 0 & $0.00 \sim 39.06$ & 0.305 & 13 & $507.81 \sim 546.88$ & 4.88 \\
\hline 1 & $39.06 \sim 78.13$ & 0.305 & 14 & $546.88 \sim 585.94$ & 4.88 \\
\hline 2 & $78.13 \sim 117.19$ & 1.22 & 15 & $585.94 \sim 625.00$ & 4.88 \\
\hline 3 & $117.19 \sim 156.25$ & 2.44 & 16 & $625.00 \sim 664.06$ & 4.88 \\
\hline 4 & $156.25 \sim 195.31$ & 4.88 & 17 & $664.06 \sim 703.13$ & 4.88 \\
\hline 5 & $195.31 \sim 234.38$ & 4.88 & 18 & $703.13 \sim 742.19$ & 4.88 \\
\hline 6 & $234.38 \sim 273.44$ & 4.88 & 19 & $742.19 \sim 781.25$ & 4.88 \\
\hline 7 & $273.44 \sim 312.50$ & 4.88 & 20 & $781.25 \sim 820.31$ & 4.88 \\
\hline 8 & $312.50 \sim 351.56$ & 4.88 & 21 & $820.31 \sim 859.38$ & 4.88 \\
\hline 9 & $351.56 \sim 390.63$ & 4.88 & 22 & $859.38 \sim 898.44$ & 4.88 \\
\hline 10 & $390.63 \sim 429.69$ & 4.88 & 23 & $898.44 \sim 937.50$ & 4.88 \\
\hline 11 & $429.69 \sim 468.75$ & 4.88 & 24 & $937.50 \sim 976.56$ & 4.88 \\
\hline 12 & $468.75 \sim 507.81$ & 4.88 & 25 & $976.56 \sim 1015.63$ & 4.88 \\
\hline
\end{tabular}

is converted into the spectral data by the DSP, using the FFT. Thus, a digital sweep frequency analyzer with very high time and frequency resolution can be realized. This technique was first implemented for the HF receiver in the PWA onboard the SS-520-2 rocket experiment (Hashimoto et al., 2003). The HF receiver onboard the SS-520-2 rocket experiment measured only 1 component of the electric field. We developed the onboard software of the WFC based on the one implemented in the PWA onboard the SS-520-2, especially for the function of a synchronization of the two PDCs because we need to determine the polarization of the wave measuring the phase difference between $\mathrm{X}$ and $\mathrm{Y}$ components.

On the other hand, the digitized data from three ADCs for the WFC-L are fed to the FIFOs (First-In, First-Out), and the DSP directly refers the data stored in the FIFOs. To determine the exact timing of the observation, the FIFO has 18-bit resolution; 16 bits are used for input data from the $\mathrm{ADC}$, and the other 2 are used for the status flags to detect the exact timing of A/D conversion. One status flag is used to synchronize with the pulse signal (1 PPS) supplied from a satellite system every second. Another flag is used for the discrimination of the transmission timing of the sounder pulse. When the SDR is in operation, a sounder pulse is transmitted every $50 \mathrm{~ms}$, and the WFC is designed to suspend its observation synchronizing with the timing of the transmission for several $\mathrm{ms}$ to avoid interference from the sounder pulse. Because of the interruption, the waveforms fed to the FIFOs become discontinuous, and this status bit stands for the interruption point of the waveform fed to the FIFOs every $50 \mathrm{~ms}$. The default interval of suspension is currently set at $4.9 \mathrm{~ms}$. This interval can be changed by setting parameters by commands and will be optimized in the phase of the first checkout of the instruments after launch. Even when the SDR is not in operation, this flag is still at work and ticks every $50 \mathrm{~ms}$. Thanks to these two status flags, we can determine the accurate timing of the data acquisition with its time resolution of less than $10 \mu \mathrm{s}$ in the data analysis on the ground.

\section{Operation Modes}

4.1 Data rates

Because of the restriction of the power consumption and telemetry budget, available observation modes of the WFC depend on the observation modes of the other LRS subsystems. The nominal data rate assigned to the LRS is $176 \mathrm{kbps}$, and $492 \mathrm{kbps}$ are also available in the special observation mode. In the $492 \mathrm{kbps}$ mode, a detailed observation for the SDR or the NPW will be performed and the WFC will be operated at the data rate of $4 \mathrm{kbps}$. In the nominal data rate mode of $176 \mathrm{kbps}$, the data rate of the WFC depends on the operation of the SDR. When the SDR is not in operation, $160 \mathrm{kbps}$ are assigned to the WFC; when the SDR is in operation, $80 \mathrm{kbps}$ are assigned to the WFC. Since the SDR uses one of two pairs of antennas as transmission antennas, another pair is available as receiving antennas. Therefore, the WFC usually uses one pair of the receiving antennas under the operation of the SDR and sends the data with a data rate of $80 \mathrm{kbps}$. Two pairs of antennas are used when the data rate of $160 \mathrm{kbps}$ are assigned to the WFC.

Because the WFC is a software receiver, both the WFC$\mathrm{H}$ and WFC-L are operated with various kinds of observation modes controlled by the DSP software. For the purpose of simplicity in the editing of the mission telemetry, the telemetry data for the WFC-H and WFC-L are independently packed into the mission packet. Because the data amount of spectrum data from the WFC-H is much smaller than that of waveform data from the WFC-L, the mission data from the WFC-H are basically sent to the ground continuously, even in a low-speed data rate mode such as $4 \mathrm{kbps}$, as long as the WFC is operated. On the other hand, the mission data from the WFC-L are nominally transported only in the high-speed data rate mode, that 
(a)
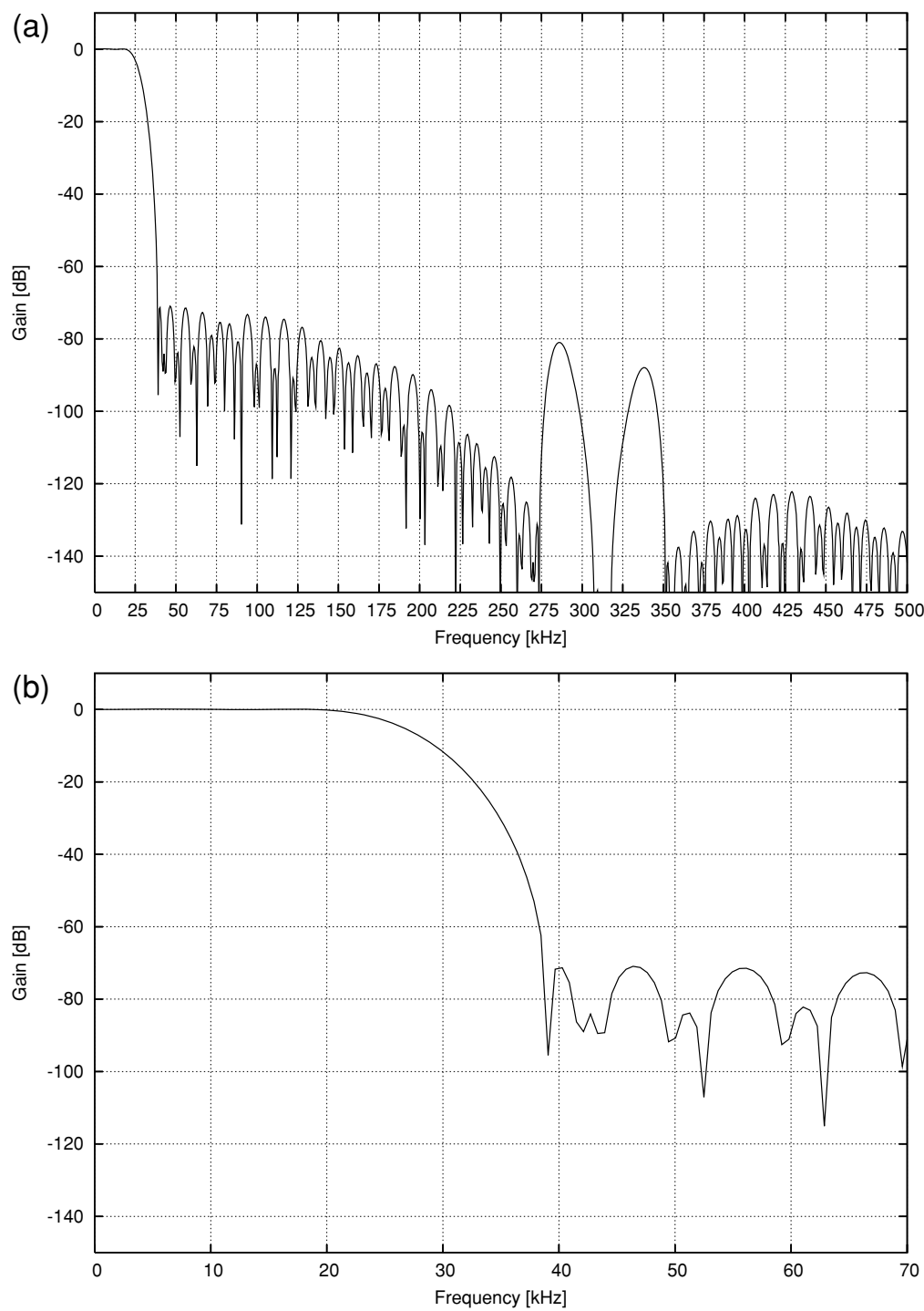

Fig. 4. (a) Composite response of the CIC, HB, and FIR filters inside the PDC below $500 \mathrm{kHz}$ when the parameters of the PDC are set for the H-mode observation. (b) The enlargement of Fig. 4(a) in the frequency range below $70 \mathrm{kHz}$.

is, if 80 or $160 \mathrm{kbps}$ are assigned to the WFC. The packet ratio between the WFC-L and WFC-H can be changed by sending parameters to the onboard software of the WFC by command. In the nominal operation, the packet ratio between the WFC-L and WFC-H is 8:2 in the $80 \mathrm{kbps}$ or 160 kbps modes and 0:10 or 5:5 in the $4 \mathrm{kbps}$ mode. In the case of the 0:10-ratio in the $4 \mathrm{kbps}$ mode, the mission packets for the WFC-L are once stored in the memory of the OBC and can be restored by a DUMP command in the high-speed data rate mode.

\subsection{WFC-H}

To cover a wide frequency range with fine time and frequency resolution, the WFC-H has two kinds of sweep mode, $\mathrm{H}$ and $\mathrm{L}$. The frequency table is shown in Table 1 . In the $\mathrm{H}$ mode, the WFC-H sweeps a whole frequency range up to $1 \mathrm{MHz}$ with a frequency step of $39.06 \mathrm{kHz}$ by changing the local frequency $f_{\mathrm{c}}$ of NCO. The decimation rates are set at 4,2 , and 4 in the CIC, HB, and FIR filters, respectively. The composite response of these filters is shown in Fig. 4. We extract the narrow band signal using this filter response by setting the local frequency of the NCO at $f_{c}=19.53+39.06 \times n \mathrm{kHz}$, where $n$ is the band number of the WFC-H shown in Table 1. It is also noted that less numbers of data are taken out from the PDC output in the higher frequency band, and the frequency resolution becomes coarser at a higher frequency range as shown in Table 1 so as to reduce the amount of telemetry data. In the L mode, two taps of HB filters are used in the PDC, and the decimation rates are set at 8,4 , and 4 in the CIC, HBs, and FIR filters, respectively. In this mode, the WFC-H covers a frequency range below $9.77 \mathrm{kHz}$ with frequency resolution of $38.2 \mathrm{~Hz}$. In the nominal operation, the $\mathrm{H}$ and $\mathrm{L}$ modes are alternately operated within 1 second, and we can obtain the spectrum data below $1 \mathrm{MHz}$ with fine time and frequency resolution.

From the point of view of the telemetry format of the WFC-H, there are three operation modes for the WFC$\mathrm{H}$ : WAVE, FFT, and PHASE. In the WAVE mode, the down-sampled data, which are the raw output data from the PDCs and consist of in-phase and quadrature components of the signal, are directly packed into the mission data. In the FFT mode, the spectrum data are processed with 
Table 2. The available duration of the continuous waveform of the WFC-L to be stored in the SRAM.

\begin{tabular}{ccc}
\hline \multirow{2}{*}{ No. of ch. } & \multicolumn{2}{c}{ Word rate $\left(f_{\mathrm{s}}\right)$ of $\mathrm{ADC}$} \\
\cline { 2 - 3 } & $125 \mathrm{kHz}$ & $250 \mathrm{kHz}$ \\
\hline $1 \mathrm{ch}: \mathrm{X} / \mathrm{Y} 1 / \mathrm{Y} 2$ & $6.0 \mathrm{~s}$ & $3.0 \mathrm{~s}$ \\
$2 \mathrm{ch}:(\mathrm{X}+\mathrm{Y} 1) /(\mathrm{Y} 1+\mathrm{Y} 2)^{*}$ & $3.0 \mathrm{~s}$ & $1.5 \mathrm{~s}$ \\
$3 \mathrm{ch}: \mathrm{X}+\mathrm{Y} 1+\mathrm{Y} 2$ & $2.0 \mathrm{~s}$ & $1.0 \mathrm{~s}$ \\
\hline
\end{tabular}

*The combination of $\mathrm{X}+\mathrm{Y} 2$ is also available as a function of the onboard software, but $\mathrm{X}+\mathrm{Y} 1$ using two pairs of dipole antennas or $\mathrm{Y} 1+\mathrm{Y} 2$ using a pair of monopole antennas will be nominally used from a scientific point of view.

the FFT by the onboard software, using the output data from the PDCs, and the intensity at each frequency point is transported to the ground. In this mode, the time resolution becomes 4 times the WAVE mode instead of sacrificing the phase data. The PHASE mode is effective when the SDR is not in operation and both components of $\mathrm{X}$ and $\mathrm{Y}$ are simultaneously obtained. In this mode, the output data from the two PDCs are processed with the FFT, and the intensity and phase difference between $\mathrm{X}$ and $\mathrm{Y}$ components at each frequency point is transported to the ground.

\subsection{WFC-L}

The WFC-L captures a few seconds of continuous waveforms intermittently. Because the data rate of the raw waveform data produced by the ADCs is far greater than the data rate capacity of the telemetry, the waveform data are intermittently acquired by the following method. The waveform data taken from the FIFOs are stored in the SRAM connected to the DSP at the first stage of the data processing routine of the WFC-L. The capacity of the SRAM for the waveform data is 1.5 megabytes; thus, 750,000 points of waveform data can be continuously stored. The onboard software in the DSP begins to store the continuous waveform data into the SRAM immediately after clearing the FIFOs until the storage area of the SRAM becomes full. These stored data are then transported to the ground over dozens of seconds. The duration of the continuous waveform depends on the output word rate of the ADCs (125 kHz or $250 \mathrm{kHz})$ and the number of channels $(1,2$ or $3)$ to be acquired. For example, if we measure only one channel among X, Y1, and Y2, 750,000 points of waveform data are continuously stored in SRAM. In the same way, 375,000 points/ch in the 2-channel mode and 250,000 points/ch in the 3-channel mode can be stored in SRAM. The available duration of the continuous waveform data to be stored in SRAM is shown in Table 2 as functions of the output word rate of the ADCs and the number of channels. In the packaging process of the data, we need to divide the waveform data into hundreds of blocks. Therefore, we put the information of the time counter (TI) of the satellite when the FIFOs are cleared, the sequence number of the divided block, and the positions of the status flags of 1PPS and 50msec ticks in the header of the mission packets. We implemented three additional functions as described below in the onboard software to maximize our scientific output under the restriction of the telemetry budget.

\section{(1) Digital filtering}

One is a digital filtering function inside the DSP. As de-

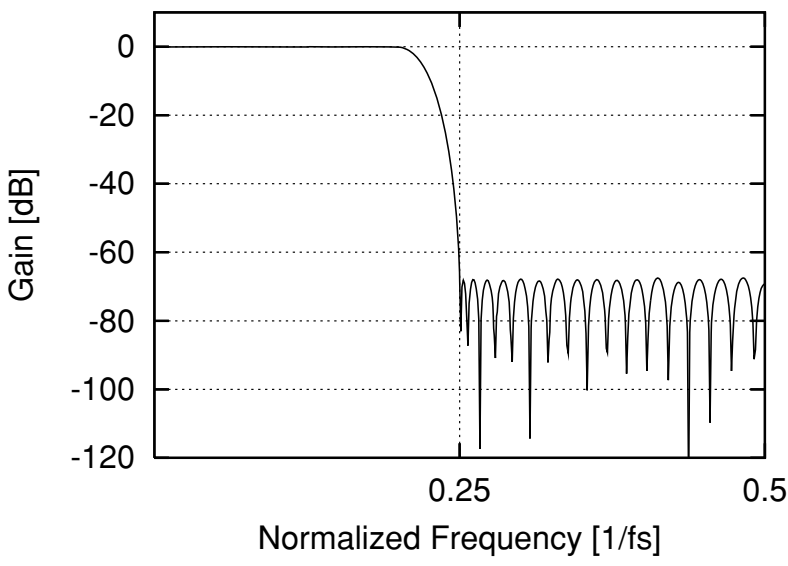

Fig. 5. Frequency response of the decimation filter implemented in the DSP for the WFC-L. Frequency is normalized by a word rate $\left(f_{\mathrm{s}}\right)$.

scribed in the previous section, the maximum frequency of the WFC-L is 50 or $100 \mathrm{kHz}$ by selecting the output word rate of the ADCs at $125 \mathrm{kHz}$ or $250 \mathrm{kHz}$. We implemented optional digital filters in the DSP. The filter is an LPF with its cutoff frequency of $1 / 4 f_{\mathrm{s}}$ and the decimation rate of 2 . The frequency response of the decimation filter is shown in Fig. 5. In the figure, the frequency in the horizontal axis is normalized by $f_{\mathrm{s}}$. This digital filter can be cascaded with 3 taps at most. When the $f_{\mathrm{s}}$ of the ADC is set at $125 \mathrm{kHz}$, for example, the maximum frequency of the waveform is lowered to $25 \mathrm{kHz}, 12.5 \mathrm{kHz}$, and $6.25 \mathrm{kHz}$, and the corresponding duty ratio (the ratio of the observation duration to the data transportation duration) can be raised by 2,4 , and 8 times, respectively.

The conceptual block diagram of the filtering system is shown in the upper part of Fig. 6. Selecting the number of taps to be used for data processing in the DSP by command, we can change the maximum frequency of the WFC-L. The relation between duty ratio and the frequency coverage of the WFC-L is shown in Table 3. In the table, we also show the time resolution of the WFC-H corresponding to each observation mode. Because the waveform observation is more important in the lower frequency range from a scientific point of view, it is essential to arrange the combination of the observation modes, an intermittent measurement covering higher frequency range with lower duty ratio and a semicontinuous measurement in the lower frequency range, to achieve the maximum scientific output.

\section{(2) Automatic filter selection}

To utilize the digital filter function more effectively, we defined a new observation mode named "automatic filter selection mode" to be realized by the onboard software. The conceptual block diagram of the filter selection system is shown in the lower part of Fig. 6. There are three kinds of band-pass filter (BPF) constructed by a digital filtering technique in the DSP software for the purpose of automatic selection. These BPFs are designed with their passband range of $25-50 \mathrm{kHz}, 12.5-50 \mathrm{kHz}$, and $6.25-50 \mathrm{kHz}$, respectively, when the output word rate $f_{\mathrm{s}}$ of the ADCs equals $125 \mathrm{kHz}$. These filters are available even at $f_{\mathrm{s}}=250 \mathrm{kHz}$, with their passband range twice those at $f_{\mathrm{s}}=125 \mathrm{kHz}$ (see Fig. 6). In the automatic filter selection mode, the waveform data 


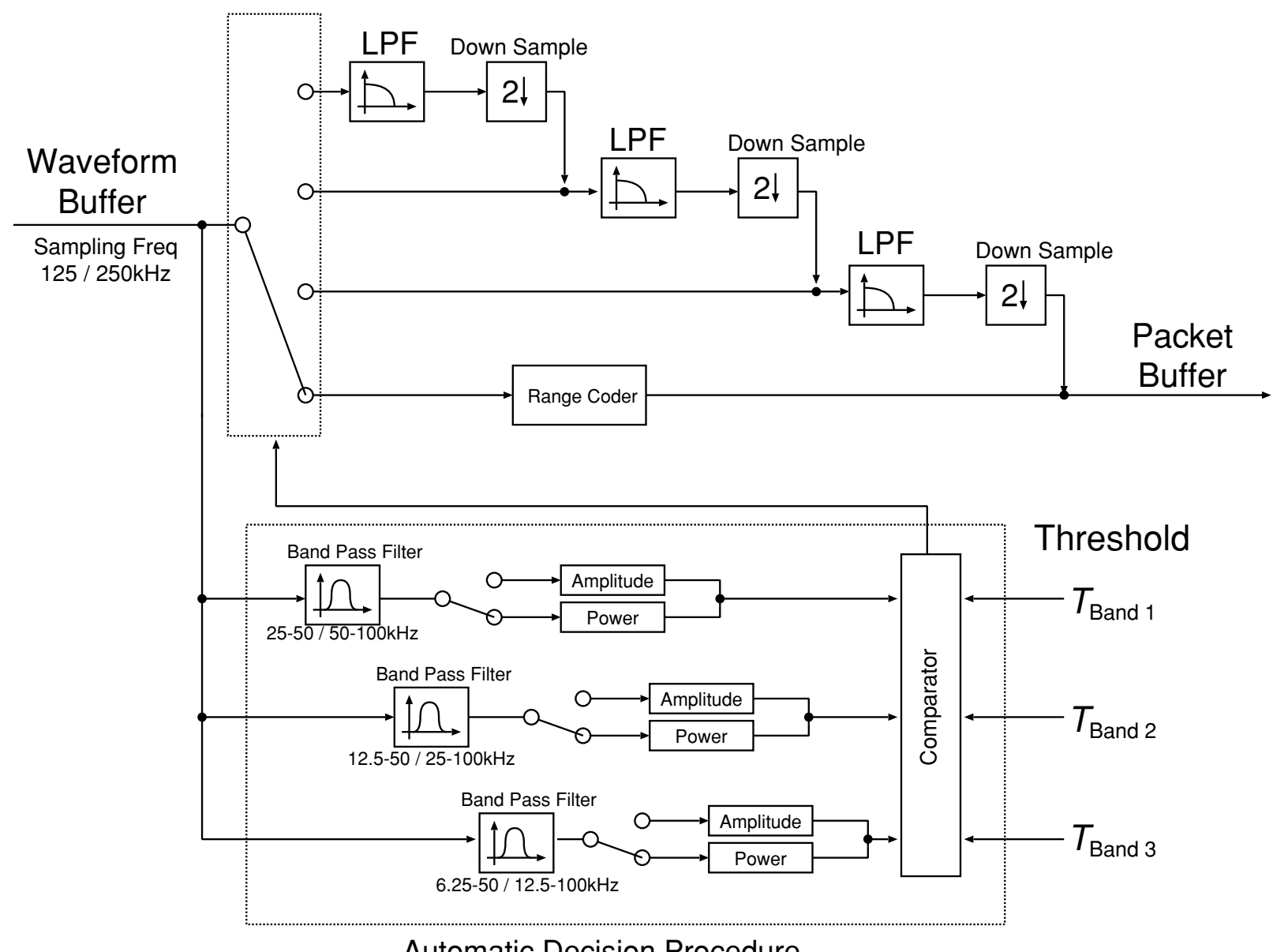

Automatic Decision Procedure

Fig. 6. A conceptual block diagram of the decimation filters and the automatic selection logic implemented in the onboard software for the WFC-L.

Table 3. A summary of the duty ratio of the WFC-L and the time resolution of the WFC-H in the nominal operation modes.

\begin{tabular}{|c|c|c|c|c|c|c|c|c|c|}
\hline \multirow[t]{2}{*}{$\begin{array}{l}\text { Bitrate } \\
{[\mathrm{kbps}]}\end{array}$} & \multirow[t]{2}{*}{ No. of ch. } & \multirow{2}{*}{$\begin{array}{c}\text { Packet } \\
\text { ratio } \\
(\mathrm{L}: \mathrm{H}) \\
\end{array}$} & \multicolumn{5}{|c|}{$\begin{array}{c}\text { Duty ratio } * *[\%] \text { vs. maximum frequency of WFC-L } \\
\left({ }^{* *} \text { without data compression }\right)\end{array}$} & \multicolumn{2}{|c|}{$\Delta t[\mathrm{~s}]$ of WFC-H } \\
\hline & & & $100 \mathrm{kHz}$ & $50 \mathrm{kHz}$ & $25 \mathrm{kHz}$ & $12.5 \mathrm{kHz}$ & $6.25 \mathrm{kHz}$ & WAVE & FFT \\
\hline \multirow[t]{2}{*}{160} & $2 \mathrm{ch}$ & $8: 2$ & 1.28 & 2.56 & 5.12 & 10.2 & 20.5 & 2 & 0.5 \\
\hline & $(1 \mathrm{ch})$ & & $(2.56)$ & $(5.12)$ & $(10.2)$ & $(20.5)$ & $(41.0)$ & (1) & $(0.25)$ \\
\hline 80 & $1 \mathrm{ch}$ & $8: 2$ & 1.28 & 2.56 & 5.12 & 10.2 & 20.5 & 2 & 0.5 \\
\hline 4 & $1 \mathrm{ch}$ & $0: 10$ & - & - & - & - & - & 8 & 2 \\
\hline
\end{tabular}

stored in SRAM are once passed through these BPFs, and the onboard software evaluates the maximum amplitudes or the averaged powers of the output signals from these filters to determine whether the waveform data in the higher frequency ranges are necessary. If the amplitudes or powers of the output signals from these BPFs are all weak, for example, the signal in only the lowest frequency range is passed through, and the waveform data packed into the mission packet are decimated with $1 / 8$ by the 3 taps of cascaded LPFs described in the previous paragraph. If any of the output signals from these BPFs are intense, the onboard software selects adequate taps of cascaded LPFs so as to involve the waveform of the corresponding frequency range. This technique makes it possible to downlink the significant waveform to the ground in a shorter time duration and to capture a new series of waveform data with a higher duty ratio. Because the threshold level for the decision at each frequency range can be changed by command, it is desirable to use this operation mode, setting adequate threshold levels after accumulating a certain amount of data and analyzing them on the ground after launch.

(3) Data compression function

The last technique implemented for the WFC-L is a data compression algorithm. In the SS-520-2 rocket experiment, a lossy compression method was adopted (Hashimoto et al., 2003). In the SELENE mission, however, we adopt a range coder that is one of lossless compression algorithms (Range Encoder Homepage, 1999). This is because the frequency range of the WFC-L onboard SELENE is much wider than that of the SS-520-2 rocket experiment $(100 \mathrm{kHz}$ for the SELENE spacecraft against $15 \mathrm{kHz}$ for the SS-520-2), and a real-time signal processing for 3 channels of waveform data from the WFC-L as well as for 2 channels of the WFC$\mathrm{H}$ is needed with one DSP chip and a restricted work area 


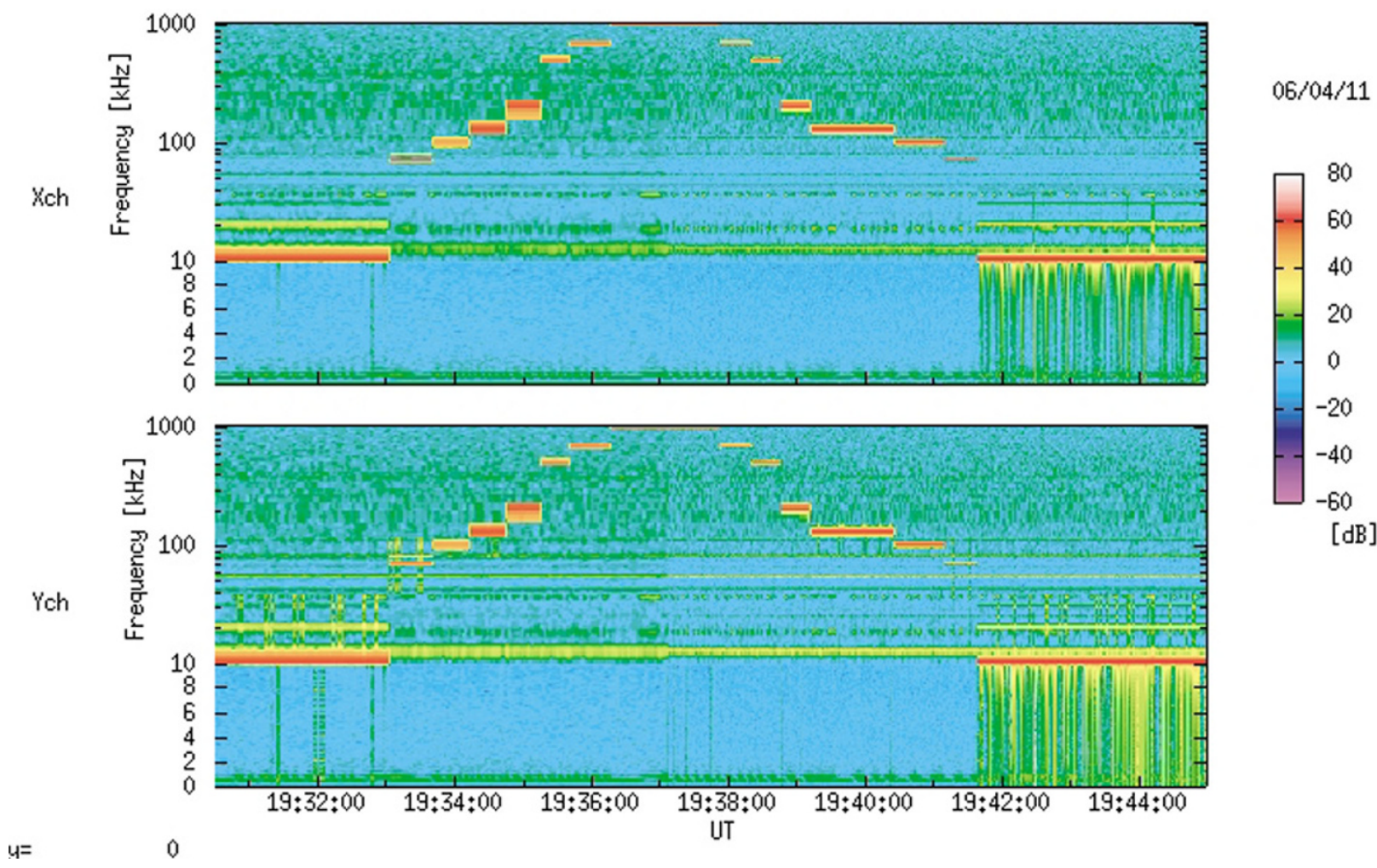

Fig. 7. An example of the spectrum diagram of the WFC-H. The upper panel shows the data from X-channel and the lower panel shows the data from Y-channel of the WFC-H. The logarithmic color scale of the intensity at each frequency point is given by the color bar at the right. The WFC-H was operated with the WAVE mode from 19:30:00 to 19:37:00, and the FFT mode from 19:37:00 to 19:45:00. Calibration signals were fed to the preamplifiers by an external function generator at $10 \mathrm{kHz}, 70 \mathrm{kHz}, 100 \mathrm{kHz}, 130 \mathrm{kHz}, 200 \mathrm{kHz}, 500 \mathrm{kHz}, 700 \mathrm{kHz}$, and $1 \mathrm{MHz}$ in that order, and in reverse order for the WAVE and FFT modes, respectively.

(2 megabytes SRAM in total). The calculation load for the lossy compression implemented in the SS-520-2 rocket experiment was too heavy, and that of the range coder is smaller; this method was suitable for the implementation of the onboard software for the WFC-L. Furthermore, lossy compression for a wide frequency range might have a bad influence on the nature of the waveform in case several kinds of wave phenomena are simultaneously observed. Although the compression ratio of the raw wave data will be $\sim 75 \%$ at most, according to our estimation, we gave priority to precision of the waveform over the compression ratio.

\section{Evaluation of the WFC}

The function tests for all observation modes were successfully performed, and the qualities of the data from both the WFC-H and WFC-L were verified. In this section, we briefly introduce examples obtained by the function test.

Figure 7 shows an example of the spectrum diagram of the WFC-H taken at Meisei Electric Co. Ltd. on April 11, 2006, for the purpose of calibration. The upper panel shows data from the X-channel, and the lower panel shows data from the Y-channel of the WFC-H. The logarithmic color scale of the intensity at each frequency point is given by the color bar at the right. The WFC-H was operated with the WAVE mode from 19:30:00 to 19:37:00, and the FFT mode from 19:37:00 to 19:45:00. Calibration signals were fed to the preamplifiers by an external function generator at $10 \mathrm{kHz}, 70 \mathrm{kHz}, 100 \mathrm{kHz}, 130 \mathrm{kHz}, 200 \mathrm{kHz}, 500 \mathrm{kHz}$, $700 \mathrm{kHz}$, and $1 \mathrm{MHz}$, in that order, and in the reverse

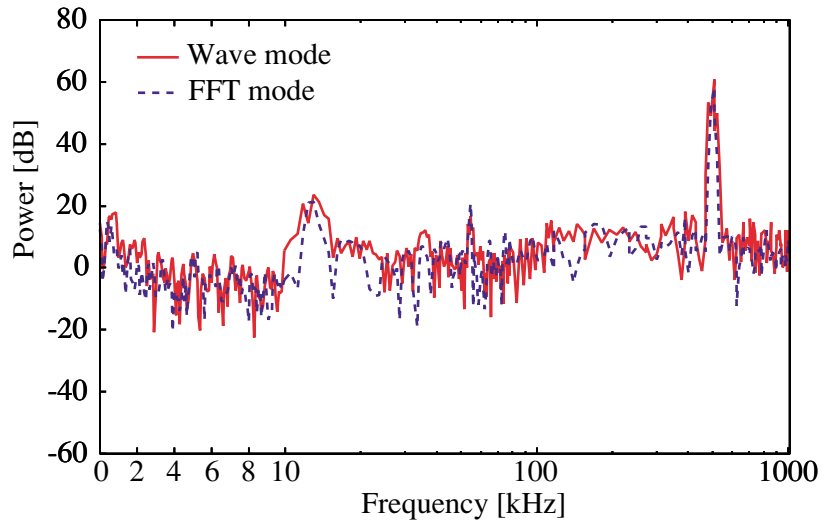

Fig. 8. A cross section of the spectrum taken by the WFC-H when fed a calibration signal at $500 \mathrm{kHz}$. The red solid line and the blue dashed line show the spectrum in the WAVE and FFT modes, respectively.

order for the WAVE and FFT modes, respectively. Figure 8 shows an example of the spectrum's cross section when a calibration signal was fed at $500 \mathrm{kHz}$. It was found that the spectral features of the WAVE and FFT modes were in good agreement with each other.

Figure 9 shows an example of a snapshot of the quicklook system for the WFC-L taken at Meisei Electric Co. Ltd. on the same date (April 11, 2006) as those shown in Fig. 7. Three panels in the upper left side are the spectrum diagrams of the WFC-L-X, WFC-L-Y1, and WFC-L$\mathrm{Y} 2$, respectively, by processing the FFT analysis by ground 


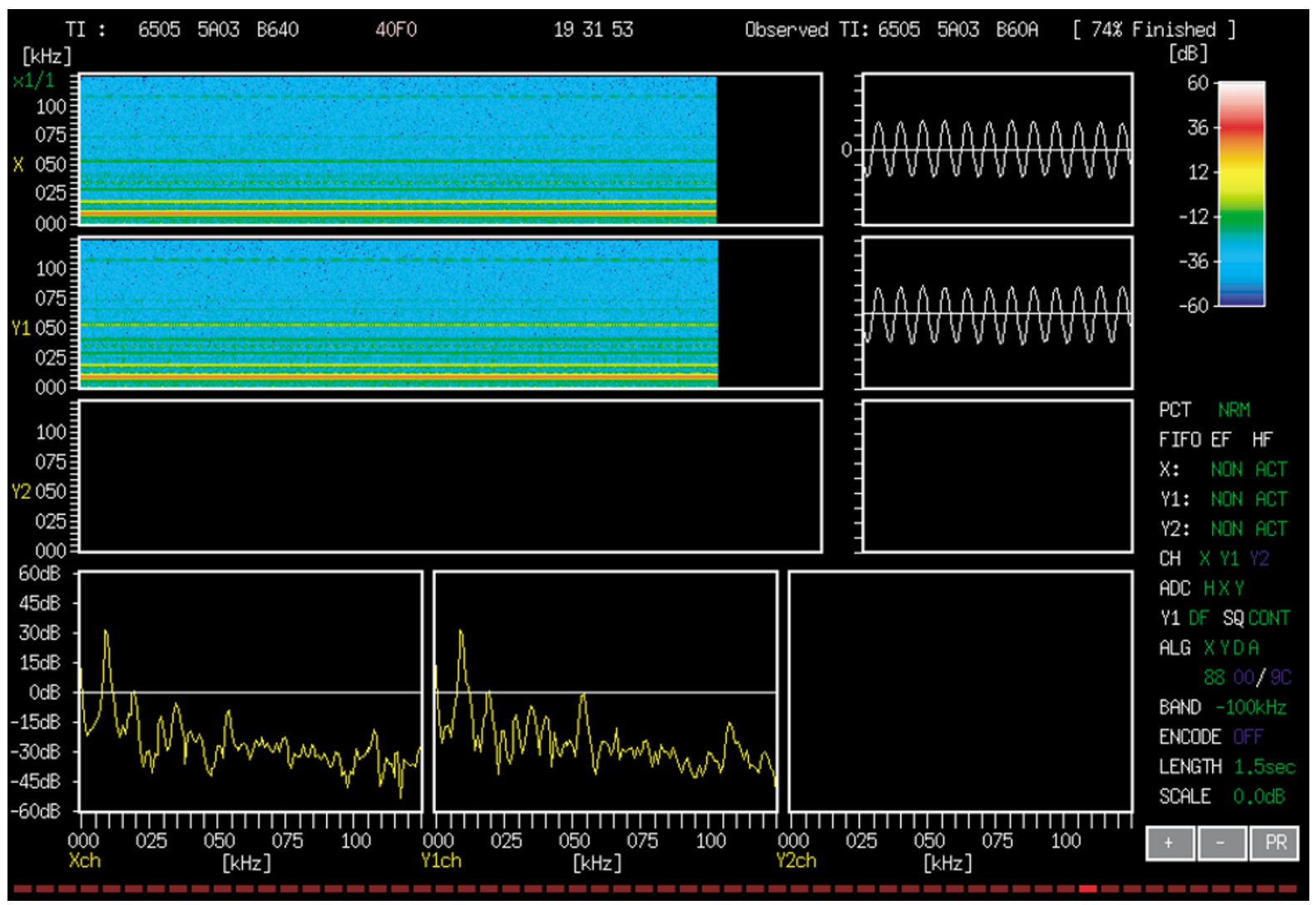

Fig. 9. An example of a snapshot of the quick-look system for the WFC-L. Three panels in the upper left side are the spectrum diagrams of the WFC-L-X, WFC-L-Y1, and WFC-L-Y2, respectively, by processing the FFT analysis using the ground support equipment. The logarithmic color scale of the intensity at each frequency point is given by the color bar at the right end. The panel on the right at the bottom shows the instantaneous waveform and cross section of the spectrum diagram corresponding to each channel. Three hundred points of waveforms are drawn in the waveform diagrams; that is, the time scale of the waveform diagrams is $1.2 \mathrm{~ms}$ or $2.4 \mathrm{~ms}$ when the output word rate $f_{\mathrm{s}}$ of the ADCs for the WFC-L equals $250 \mathrm{kHz}$ or $125 \mathrm{kHz}$, respectively. In this instance, the WFC-L was operated in the 2-channel mode, taking the waveforms from the WFC-L-X and WFC-L-Y1 (X and Y1 in CH are displayed in green and Y2 in blue) with $f_{\mathrm{s}}=250 \mathrm{kHz}$ (BAND is up to $-100 \mathrm{kHz}$ ) setting the multiplexer of the WFC-L-Y1 at the differential signal mode (Y1 is DF). A calibration signal at $10 \mathrm{kHz}$ was fed to both of the WFC-L-X and WFC-L-Y1 channels by an external function generator.

support equipment, using the mission packets of the WFCL. The logarithmic color scale of the intensity at each frequency point is given by the color bar at the right end. This quick-look system is used to briefly check out the instruments. These spectrum diagrams are drawn from left to right repeatedly, and the right end of the color contour in the diagrams show the drawing's current position. The time of the waveform is shown at the top line on the right side as "Observed TI" with a 12-digit hexadecimal number (6505 $5 \mathrm{~A} 03 \mathrm{~B} 60 \mathrm{~A})$, which is the time counter of the satellite when the FIFOs of WFC-L are cleared. "XX \% Finished" represents the ratio of the already downlinked waveform data to the stored waveform data in the SRAM. The time when the mission packet was produced is shown at the top line on the left side as "TI" with a 12-digit hexadecimal number (6505 5 A03 B640). The universal time (UT) is also shown as "19 31 53", which is the time when the mission packet was received at the ground station. In the nominal operation after launch, a conversion table between TI and UT will be delivered by the SELENE project team, and we need to convert observed TI to UT for detailed data analysis. Three panels on the right of the spectrum diagrams show an instantaneous waveform of the current drawing in the spectrum diagrams for WFC-L-X, WFC-L-Y1, and WFC-L-Y2, respectively.
A total of 300 points of waveforms are drawn in the waveform diagrams; that is, the time scale of the waveform diagrams is $1.2 \mathrm{~ms}$ or $2.4 \mathrm{~ms}$ when the output word rate $f_{\mathrm{s}}$ of the ADCs for WFC-L equals $250 \mathrm{kHz}$ or $125 \mathrm{kHz}$, respectively. In the same way, the three panels at the bottom show the instantaneous cross sections of the spectrum diagrams for WFC-L-X, WFC-L-Y1, and WFC-L-Y2, respectively.

There are many housekeeping (HK) status data, which show the conditions of the instruments, and important HK data related to the WFC are shown in the right side. At this time, for example, the WFC-L was operated in the 2-channel mode, taking the waveforms from the WFC-L$\mathrm{X}$ and WFC-L-Y1 ( $\mathrm{X}$ and $\mathrm{Y} 1$ in $\mathrm{CH}$ were displayed in green and Y2 in blue) with $f_{\mathrm{s}}=250 \mathrm{kHz}$ (BAND is up to $-100 \mathrm{kHz}$ ) setting the multiplexer of the WFC-L-Y1 at the differential signal mode (Y1 is DF).

A calibration signal at $10 \mathrm{kHz}$ was fed into both WFC-L$\mathrm{X}$ and WFC-L-Y1 channels by an external function generator, and the signals are clearly seen in both channels. Because the WFC-L-Y2 channel was turned off, the data for this channel are not displayed. Clear peaks at $10 \mathrm{kHz}$ in the spectrum diagrams (upper left panels) and cross sections (bottom panels) are visible. In addition, 12-cycle waveforms in the waveform diagrams (with their time scale of 
$1.2 \mathrm{~ms}$ as described in the previous paragraph), which indicate the $10 \mathrm{kHz}$ signal, are visible. Although the data quality is roughly shown by this quick-look system, we evaluated it in more detail and confirmed that the signal level and signal-to-noise ratio are in satisfactory and that the phase difference between the two channels is negligible $(\sim 0.5 \mu \mathrm{s})$.

\section{Summary}

We developed a high-performance and multifunctional software receiver named WFC for the measurement of plasma waves and radio emissions on board SELENE. To perform spectral analysis with very high time and frequency resolution covering a wide frequency range from $1 \mathrm{kHz}$ to $1 \mathrm{MHz}$, a hybrid IC called PDC was introduced into the WFC-H. For the lower frequency range below $100 \mathrm{kHz}$, we developed an intelligent receiver named WFC-L to measure waveforms with high-duty ratio. New techniques such as digital filtering, automatic filter selection, and data compression are additionally implemented on the onboard software to extract important data adequately under the severe restrictions on the total amount of telemetry data.

The basic data for the calibration were cautiously obtained, and the function test for the instrument was also successfully performed. Because of the flexibility of the instruments, various kinds of observation modes can be achieved, and we expect the WFC to produce many interesting scientific data.

Acknowledgments. The SELENE project has been organized by the Japan Aerospace Exploration Agency (JAXA). The authors express their deep appreciation to Drs. H. Kojima and Y. Ueda and to Mr. H. Iwai for their valuable suggestions and discussions on the development of the onboard software. The authors are also grateful to Professors I. Nagano, T. Okada, and M. Tsutsui and to Drs. Y. Kasaba, S. Yagitani, and K. Ishisaka for their helpful suggestions and contribution to the hardware design and the development of the WFC. The authors thank Mr. A. Muro for his help in the evaluation of the data quality of the WFC. The LRSWFC hardware was manufactured by Meisei Electric Company. The authors express their thanks to all members of the SELENE project team.

\section{References}

Analog Devices Inc., AD9260 data sheet, 2000.

Anderson, R. R., D. A. Gurnett, H. Matsumoto, K. Hashimoto, H. Kojima, Y. Kasaba, M. L. Kaiser, G. Rostoker, J.-L. Bougeret, J.-L. Steinberg, I. Nagano, and H. Singer, Observations of low frequency terrestrial type III bursts by GEOTAIL and WIND and their association with isolated geomagnetic disturbances detected by ground and space-borne instruments, in Planetary Radio Emissions IV, Proc. Graz Conf., edited by H. O. Rucker, S. J. Bauer, and A. Lecacheux, Austrian Academy of Sciences Press, Vienna, 241-250, 1997.

Bougeret, J.-L., M. L. Kaiser, P. J. Kellogg, R. Manning, K. Goetz, S. J. Monson, N. Monge, L. Friel, C. A. Meetre, C. Perche, L. Sitruk, and S. Hoang, WAVES: The radio and plasma wave investigation on the wind spacecraft, Space Sci. Rev., 71, 231-263, 1995.

Farrell, W. M., M. L. Kaiser, and J. T. Steinberg, Electrostatic instability in the central lunar wake: A process for replenishing the plasma void?, Geophys. Res. Lett., 24(9), 1135-1138, 1997.

Hashimoto, K., H. Matsumoto, T. Murata, M. L. Kaiser, and J.-L. Bougeret, Comparison of AKR simultaneously observed by the GEOTAIL and WIND spacecraft, Geophys. Res. Lett., 25(6), 853-856, 1998.

Hashimoto, K., W. Calvert, and H. Matsumoto, Kilometric continuum detected by GEOTAIL, J. Geophys. Res., 104(A12), 28,645-28,656, 1999.

Hashimoto, K., H. Iwai, Y. Ueda, H. Kojima, and H. Matsumoto, Software wave receiver for the SS-520-2 rocket experiment, IEEE Trans. Geosci. and Remote Sens., 41(11), 2638-2647, 2003.

Hashimoto, K., R. R. Anderson, J. L. Green, and H. Matsumoto, Source and propagation characteristics of kilometric continuum observed with multiple satellites, J. Geophys. Res., 110(A09229), doi: 10.1029/2004JA010729, 2005.

Hashimoto, K., J. L. Green, R. R. Anderson, and H. Matsumoto, Review of Kilometric Continuum, Lecture Notes in Physics, Springer-Verlag, Berlin Heidelberg, 687, 37-54, 2006.

Intersil Corporation, HSP50214B data sheet, 2000.

Kasaba, Y., H. Kojima, H. Matsumoto, and T. Murata, Search for Jovian Hectometric and Kilometric radiation by GEOTAIL spacecraft during the impact of Comet Shoemaker-Levy 9, J. Geomag. Geoelectr., 48(4), 361-370, 1996.

Kasaba, Y., H. Matsumoto, K. Hashimoto, R. R. Anderson, J.-L. Bougeret, M. L. Kaiser, and X. Y. Wu, Remote sensing of the plasmapause during substorms: GEOTAIL observation of nonthermal continuum enhancement, J. Geophys. Res., 103(A9), 20,389-20,405, 1998.

Kasaba, Y., H. Matsumoto, Y. Omura, R. R. Anderson, T. Mukai, Y. Saito, T. Yamamoto, and S. Kokubun, Statistical studies of plasma waves and backstreaming electrons in the terrestrial electron foreshock observed by GEOTAIL, J. Geophys. Res., 105(A1), 79-103, 2000.

Kasahara, Y., H. Matsumoto, and H. Kojima, Type III radio bursts in the inhomogeneous interplanetary space observed by GEOTAIL, Radio Sci., 36(6), 1701-1711, 2001.

Kellogg, P. J., K. Goetz, and S. J. Monson, Observations of plasma waves during a traversal of the moon's wake, Geophys. Res. Lett., 23(10), 1267-1279, 1996.

Kojima, H., M. Ashour-Abdalla, W. R. Paterson, H. Matsumoto, L. A. Frank, R. R. Anderson, R. L. Richard, S. Kokubun, and T. Yamamoto, Generation of the narrowband electrostatic noise in the geomagnetic tail: GEOTAIL observations, J. Geophys. Res., 106(A5), 8483-8488, 2001.

Kuncic, Z. and I. H. Cairns, Radio emission from mini-magnetospheres on the moon, Geophys. Res. Lett., 31(L11809), doi:10.1029/2004GL 020008, 2004.

Kurata, M., H. Tsunakawa, Y. Saito, H. Shibuya, M. Matsushima, and H. Shimizu, Mini-magnetosphere over the Reiner Gamma magnetic anomaly region on the moon, Geophys. Res. Lett., 32(L24205), doi: 10.1029/2005GL024097, 2005.

Mangeney, A., C. Salem, C. Lacombe, J.-L. Bougeret, C. Perche, R. Manning, P. J. Kellogg, K. Goetz, S. J. Monson, and J.-M. Bosqued, WIND observations of coherent electrostatic waves in the solar wind, Ann. Geophysicae, 17(3), 307-320, 1999.

Matsumoto, H., I. Nagano, R. R. Anderson, H. Kojima, K. Hashimoto, M. Tsutsui, T. Okada, I. Kimura, Y. Omura, and M. Okada, Plasma wave observations with GEOTAIL spacecraft, J. Geomag. Geoelectr., 46(1), 59-95, 1994a.

Matsumoto, H., H. Kojima, T. Miyatake, Y. Omura, M. Okada, I. Nagano, and M. Tsutsui, Electrostatic solitary waves (ESW) in the Magnetotail: BEN wave forms observed by GEOTAIL, Geophys. Res. Lett., 21(25), 2915-2918, 1994b.

Matsumoto, H., T. Okada, K. Hashimoto, I. Nagano, S. Yagitani, M. Tsutsui, K. Kasaba, K. Tsuruda, H. Hayakawa, A. Matsuoka, S. Watanabe, H. Ueda, I. Kimura, Y. Kasahara, Y. Omura, M. Matsumura, T. Imachi, K. Ishisaka, and Y. Tateno, Low frequency plasma wave analyzer (LFA) onboard the PLANET-B spacecraft, Earth Planets Space, 50(3), 223228, 1998.

Murata, T., H. Matsumoto, H. Kojima, and T. Iyemori, Correlations of AKR index with Kp and Dst indices, Proc. of the NIPR Symposium on Upper Atmosphere Physics, 10, 64-68, 1997.

Ono, T., A. Kumamoto, Y. Yamaguchi, A. Yamaji, T. Kobayashi, Y. Kasahara, and H. Oya, Instrumentation and observation target of the Lunar Radar Sounder (LRS) experiment on-board the SELENE spacecraft, Earth Planets Space, 60, this issue, 321-332, 2008.

Range Encoder Homepage, http://www.compressconsult.com/rangecoder/, 1999.

SELENE Project, http://www.kaguya.jaxa.jp/en/index.htm, 2007.

Texas Instruments Inc., SMJ320C31 data sheet, 2006.

Trávníček, P., P. Hellinger, D. Schriver, and S. D. Bale, Structure of the lunar wake: Two-dimensional global hybrid simulations, Geophys. Res. Lett., 32(L06102), doi:10.1029/2004GL022243, 2005.

Y. Kasahara (e-mail: kasahara@is.t.kanazawa-u.ac.jp), Y. Goto, K. Hashimoto, T. Imachi, A. Kumamoto, T. Ono, and H. Matsumoto 